\title{
Rotavirus and adenovirus in Rondônia
}

\section{Gleiciene Félix Magalhães/*, Paulo Afonso Nogueira/*, Andréa Fagundes Grava/*, Marilene Penati**, Luiz Hildebrando Pereira da Silva, Patricia Puccinelli Orlandi/*/+}

\author{
Centro de Pesquisa em Medicina Tropical, Porto Velho, RO, Brasil *Centro de Pesquisa Leônidas e Maria Deane-Fiocruz, \\ Rua Teresina 476, 69057-000 Manaus, AM, Brasil **Hospital Infantil Cosme Damião, Porto Velho, RO, Brasil
}

Acute gastroenteritis is one of the most common diseases in humans worldwide. Viral gastroenteritis is a global problem in infants and young children. In this study the incidence of diarrhea was assessed in 877 hospitalized children under five years old, over a period of 24 months and distributed in 470 cases of diarrhea and 407 age-matched group with other pathologies, as control group. Two antigen detection techniques based on enzyme immunoassay (EIA) and latex particles were used for detection of rotavirus and adenovirus. Rotavirus A was a major cause of gastroenteritis with $23.6 \%$ of cases, being $90 \%$ of these cases in young children. Adenovirus infections was detected by EIA with frequency of 6.4\%. Rotavirus and adenovirus were detected in 10.1 and $1.7 \%$ of stools from control group, respectively. Interestingly, the frequency of the youngest children in the control group excreting Rotavirus A was comparable to that detected in stools from diarrheic children. We cannot rule out the existence of other enteric viruses because the etiology of 171 cases of diarrhea was not determined and active search for astrovirus and calicivirus was not done. This is the first study that shows the presence of enteric viruses in the infantile population from Western Brazilian Amazonia and it was important to help physicians in the treatment of viral gastroenteritis.

Key words: diarrhea - rotavirus - adenovirus - enzyme immunoassay for rotavirus and adenovirus - latex - Rondônia - Brazil

Acute gastroenteritis is one the most common diseases in humans and continues to be a significant cause of morbidity and mortality worldwide. It accounts for nearly $20-40 \%$ of hospital admissions with vomiting and diarrhea followed by severe dehydration in very young children below two years old, being rotavirus a major cause of these severe gastroenteritis (WHO 1999). In developing countries rotavirus gastroenteritis is a major cause of childhood death and is responsible for approximately half a million deaths per year among children aged < five years (Parashar et al. 2006).

In Brazil, frequencies of rotavirus infection between $20-70 \%$ have been reported especially in younger children (Santos et al. 2002, Luz et al. 2005, Carvalho-Costa et al. 2006).

Rotaviruses are members of the Reoviridae family and according to the antigenic properties of the group reactivity determinant VP6 capsid protein, they are classified into seven groups, A to G, and two subgroups I and II. Groups A, B, and C are those which have been associated with infection in humans. Rotavirus from group $\mathrm{A}$ is the more prevalent cause of viral gastroenteritis and can be detected by a variety of techniques including latex agglutination (LA) and enzyme immunoassay (EIA).

\footnotetext{
Financial support: $\mathrm{CNPq}$

${ }^{+}$Corresponding author: patricia_orlandi@amazonia.fiocruz.br Received 26 October 2006

Accepted 11 June 2007
}

Human adenoviruses belong to the Adenoviridae family. The 51 different serotypes of human adenoviruses have been classified into six species (A-F) on the basis of biophysical and biochemical criteria (Benko \& Harrach 2003).

The enteric serotypes that are most frequently associated with gastroenteritis caused by adenovirus are 40 and 41, which belong to subgenus $A$ and serotypes 1, 2, 5 , and 6 of subgenus $C$ have been involved in the etiology of acute diarrhea (Baum 2000).

Enteric adenoviruses present variable incidences of infection. In industrialized countries, the incidence varies from 1 to $8 \%$, whereas in developing countries figures of 2-31\% have been published (Qiao et al. 1990).

The present study was performed in a pediatric Public Hospital from Porto Velho, capital of the state of Rondônia, located in the Western Brazilian Amazonia. The occurrence of acute gastroenteritis and prevalence of Rotavirus $A$ and adenovirus were investigated in hospitalized children under five years old.

\section{MATERIALS AND METHODS}

Study area and patients - Porto Velho is located on the right bank of the Madeira River $\left(08^{\circ} 45^{\prime} \mathrm{S}\right.$ and $63^{\circ}$ $\left.55^{\prime} \mathrm{W}\right)$. The population is estimated in 300 thousand, the majority of them living under poor sanitation condition. The "Cosme Damião" Infantile Public Hospital is a major hospital that provides assistance to the population of children. Over a period of two years, March 2000 to March 2002, stool specimens of children under five years old, who were assisted at Cosme Damião presenting with acute diarrhea and requiring hospitalization, were investigated. Diarrheal cases were defined as episodes with three or more loose stools within the previous 24 h-period. Children presenting with diarrhea lasting more than five days were excluded of this study. Chil- 
dren who were assisted at the hospital with clinical conditions other than gastroenteritis were invited to participate as control group. This group was age, sex, and economic status matched by a control, non-diarrheic group including children who had not received antibiotic therapy during the previous two weeks. This study was approved by the local ethic committees and stools specimens were collected after parents had given informed consent 463/CEP.

Stool processing - Stool specimens were collected after natural evacuation or through stimulation with glycerin suppository. The samples were concentrated by centrifugation 1:1 in Hank's complete balanced salt solution at $3500 \mathrm{~g}$ for $15 \mathrm{~min}$ at room temperature. The supernatant was centrifuged at $3500 \mathrm{~g}$ for $1 \mathrm{~h}$ at $4^{\circ} \mathrm{C}$. The pellet was solved in $200 \mu \mathrm{l}$ of $10 \mathrm{mM}$ Tris $\mathrm{HCl}$ and $\mathrm{CaCl}_{2}$ $1.5 \mathrm{mM}, \mathrm{pH} 7.2$ buffer. The extracts were stood at $-70^{\circ} \mathrm{C}$ until testing by LA and EIA methods.

Search for rotavirus and adenovirus - Brief, $60 \mu \mathrm{l}$ of clarified extracts from 877 stools specimens were tested in duplicate according to standard methods using a commercial enzyme-linked immunoassay kit for rotavirus and adenovirus detection (EIARA KitBiomanguinhos-Fiocruz) and optical densities were measured at $450 \mathrm{~nm}$ Multiscan MCC/340 Titertek Elisa (Uniscience). Search for rotavirus was confirmed by LA slidex kit of Richmond (Rich Diagnostics, New Brunswick, NJ, US) in which $50 \mu 1$ of clarified fecal extract were used with the same 877 specimens, according to standard protocols.

\section{RESULTS}

Over the period of March 2000 to 2002 the etiology of acute gastroenteritis was investigated in Cosme Damião Hospital. The distribution of 470 children by age in months is shown in the Table. As expected, children aged less than 24 months of age were those who mostly required hospitalization by gastroenteritis: 408 of 470 children (86\%). During this study eight children died of gastroenteritis.

Major enteropathogenic, bacteria diarrheogenic Escherichia coli, Salmonella sp., Shigella sp., and Yersinia enterocolitica were responsible for 158 of cases of diarrhea. The major intestinal parasites Giardia lamblia and Entamoeba histolytica were responsible for others 16 cases. These data are shown in our other study (Orlandi et al. 2006).
Search for rotavirus was assessed by EIA to rotavirus and LA kit. Percentage of agreement of positive samples observed in both tests was high. The percentage of agreement observed in both standard methods for rotavirus detection was $88.2 \%$. We found rotavirus in $111(23.6 \%)$ of 470 specimens, thus representing the main cause of diarrhea. Ninety percent of rotavirus positive stools (101/111) were collected of young children below 24 months old. Stratifying samples by month of age, the $0-6,7-12$, and 13-24 groups of diarrheic subjects had similar frequencies of rotavirus (Table), confirming what has been observed worldwide, in that rotavirus gastroenteritis is very common in children of these age groups.

A group of 407 age-matched children who presented with clinical conditions other than gastroenteritis were invited to participate as control group. Rotavirus was found with a frequency of $10.1 \%$, and alike diarrhea group, rotavirus was prevalent in younger children of control (Table). This frequency of the youngest children in the control group excreting rotavirus was comparable to that of the diarrheic children.

Adenovirus gastroenteritis was investigated by EIA and observed frequency was $6.4 \%$. Adenovirus was found in $1.7 \%$ of stools of control group.

\section{DISCUSSION}

Acute gastroenteritis is one of major the causes of illness in infantile population worldwide. Children under five years old are particularly prone to suffer from gastroenteritis every year and in general their symptoms require hospitalization. Rotavirus $A$ is the main responsible for cases that occur in general among young children and their symptoms may often require hospitalization. Several European studies point to Rotavirus A as the agent responsible for 20-60\% of cases of gastroenteritis (Whilhelmi et al. 2003). In Australia, similar figures have been reported (Barnez et al. 1998) and in the United States it has been estimated that one in every 73 children will be hospitalized because of diarrhea due to rotavirus during the first five years of life (Glass 1996).

In Brazil, several studies have reported frequencies of rotavirus infection between $20-70 \%$, especially in younger children (Santos et al. 2002, Luz et al. 2005, Carvalho-Costa et al. 2006). Our results are comparable to those from large cities of different states which showed the occurrence of rotavirus at a rate of approximately $23 \%$ among infants hospitalized for acute diar-

TABLE

Distribution of groups by months of age and frequency of positives in rotavirus and adenovirus search

\begin{tabular}{|c|c|c|c|c|c|c|}
\hline \multirow{2}{*}{$\begin{array}{l}\text { Distribution of groups } \\
\text { by months of age }\end{array}$} & \multirow[b]{2}{*}{$\mathrm{N}$} & \multicolumn{2}{|c|}{$\begin{array}{c}\text { Diarrhea group } \\
\text { Number of positive (Frequency \%) }\end{array}$} & \multirow[b]{2}{*}{$\mathrm{N}$} & \multicolumn{2}{|c|}{$\begin{array}{c}\text { Control group } \\
\text { Number of positive (Frequency \%) }\end{array}$} \\
\hline & & Rotavirus & Adenovirus & & Rotavirus & Adenovirus \\
\hline $0-6$ & 107 & $22(20.6)$ & $6(5.6)$ & 84 & $12(14.3)$ & $1(1.2)$ \\
\hline $7-12$ & 144 & $34(23.6)$ & $12(8.3)$ & 97 & $12(12.4)$ & $2(2.1)$ \\
\hline $13-24$ & 157 & 45 (28.7) & $12(7.6)$ & 152 & $13(8.6)$ & $3(2.0)$ \\
\hline $25-60$ & 62 & $10(16.1)$ & $0(0)$ & 74 & $4(5.4)$ & $1(1.4)$ \\
\hline All & 470 & 111 (23.6) & $30(6.4)$ & 407 & $41(10.1)$ & $7(1.7)$ \\
\hline
\end{tabular}


rhea. Interestingly, our data also show that an unusual high proportion $(\sim 10 \%)$ of hospitalized infants without diarrhea were excreting rotavirus, as observed in other poor areas in Brazil (Luz et al. 2005). For some as yet unexplained reason rotaviruses appeared to have spread efficiently within pediatric wards, probably causing asymptomatic nosocomial infection in a significant proportion of children.

This study added several data to the knowledge on the epidemiology of rotavirus infection in one of the country's poorest region where poverty and poor sanitation conditions largely prevail among both periurban and rural communities.

Adenoviruses occurred at variable rates of infection. In developing countries the incidence varies from 2-31\% (Wilhelmi et al. 2003). In this study, the frequency of adenovirus was $6.4 \%$. Orlandi et al. (2006) report that rotavirus infection represents the main cause of gastroenteritis. Of 470 children from the diarrhea group, Rotavirus $A$ and adenovirus were responsible for 141 cases of diarrhea. The etiology of others 174 cases of diarrhea is shown in other study, at which major enteropathogenic of bacteria and protozoa groups were responsible for them (Orlandi et al. 2006). However in 155 cases of diarrhea the etiology could not be determined. As an active search for astrovirus and calicivirus was not done in this study, the overall incidence of viral gastroenteritis can be higher than currently observed.

Although the present study has added epidemiological data on rotavirus infections to those of the more Northern tropical areas of Brazil, a further characterization of strains recovered from hospitalized children in North region is worth to be pursued. This would comprise a broader characterization of rotavirus genotypes by use of reverse transcriptase polymerase chain reaction.

This is the first study that investigated the presence of enteric viruses in an infantile population from Western Brazilian Amazonia. Testing for rotavirus unfortunately is not performed on a routine basis when a diarrheic child seeks for medical care. This study was important to make physicians aware of the importance of viral gastroenteritis in Porto Velho, Rondônia.

\section{REFERENCES}

Barnez GL, Uren U, Stevens KB, Bishop RF. 1998. Etiology of acute gastroenteritis in hospitalized children in Melbourne, Australia from April 1980 to March 1993. J Clin Microbiol 36: $133-138$.

Baum SG 2000. Adenovirus. In GL Mandell, JE Bennet, R Dolin, Principles and Practice of Infectious Diseases, Churchill Livingstone, New York, p. 1624-1630.

Benkö M, Harrach B 2003. Molecular evolution of adenoviruses. Curr Top Microbiol Immunol 272: 3-35.

Carvalho-Costa FA, Assis RM, Fialho AM, Bóia MN, Alves DPD, Martins CMMA, Gagliardi Leite JP 2006. Detection and molecular characterization of group A rotavirus from hospitalized children in Rio de Janeiro, Brazil, 2004. Mem Inst Oswaldo Cruz 101: 291-294.

Glass RI, Kilgore PE, Holman RC 1996. The epidemiology of rotavirus diarrhea in the United States: surveillance and estimates of disease burden. J Infect Dis 174 (Suppl. 1): S5-11.

Luz CRNE, Joana D'Arc P, Mascarenhas JDP, Gabbay YB, Motta ARB, Lima TVR, Soares LS, Linhares AC 2005. Rotavirus serotypes and electropherotypes identified among hospitalized children in São Luis, Maranhão, Brazil. Rev Inst Med Trop São Paulo 47: 287-293.

Orlandi PP, Magalhães GF, Matos NB, Silva T, Penatti M, Nogueira PA, Pereira da Silva LH 2006. Etiology of diarrheal infections in children of Porto Velho (Rondônia, Western Amazon region, Brazil). Braz J Med Biol Res 39: 507-517.

Parashar UD, Alexander JP, Glass RI 2006. Prevention of rotavirus gastroenteritis among infants and children. Recommendations and reports. CDC-MMWR 55 (RR12): 1-13.

Qiao H, Nilsson M, Abreu ER 1999. Viral diarrhea in children in Beijing, China. J Med Virol 57: 390-396.

Santos N, Volotão EM, Soares CC, Campos GS, Sardi SI, Hoshino Y 2002. Predominance of rotavirus genotype G9 during the 1999, 2000, and 2002 seasons among hospitalized children in the city of Salvador, Bahia, Brazil: Implications for future vaccine strategies. J Clin Microbiol 43: 4064-4069.

Wilhelmi I, Roman E, Sachez-Fauquier A 2003. Virus causing gastroenteritis. Clin Microbiol Infect 9: 247-262.

WHO-World Health Organization 1999. Rotavirus vaccines. WHO position paper. Weekly Epidemiol Rec 74: 33-40. 
